\title{
TEOR E ACÚMULO DE NUTRIENTES EM FOLHAS E FRUTOS DE PINHÃO-MANSO ${ }^{(1)}$
}

\author{
Bruno Galvêas Laviola ${ }^{(2)}$ \& Luiz Antonio dos Santos Dias ${ }^{(3)}$
}

\begin{abstract}
RESUMO
O conhecimento das necessidades nutricionais de uma cultura é essencial para a elaboração das recomendações de adubação. De posse disso, estudou-se o teor e o acúmulo de nutrientes em folhas e frutos de pinhão-manso, bem como estimou-se a extração de nutrientes pela colheita de frutos entre o primeiro e o quarto ano de cultivo. O estudo foi realizado na fazenda Paraíso, no município de Viçosa-MG. Utilizou-se para coleta das amostras um talhão com 6.000 plantas de pinhão-manso, implantado em abril/2006, no espaçamento de 4 x $2 \mathrm{~m}$. Foi realizada a coleta aleatória de quatro amostras de folhas e de frutos na área total, sendo cada amostra composta por 15 folhas ou 20 frutos. Para as amostras de folhas, foram coletados limbos foliares expandidos, entre a sexta e a oitava folha abaixo da inflorescência. Somente folhas sem deficiência nutricional ou ataque de pragas e, ou, doenças aparentes foram amostradas. Os frutos foram coletados no estádio maduro, com a casca amarela. Os materiais vegetais amostrados foram lavados, postos a secar, pesados e submetidos às análises químicas. As folhas de pinhãomanso apresentaram a seguinte ordem de acúmulo de nutrientes: $\mathrm{N}>\mathrm{Ca}>\mathrm{K}>\mathrm{Mg}$ $>\mathrm{P}>\mathrm{S}>\mathrm{Mn}>\mathrm{Fe}>\mathrm{B}>\mathrm{Zn}>\mathrm{Cu}$; nos frutos, a ordem encontrada foi: $\mathrm{N}>\mathrm{K}>\mathrm{Ca}>\mathrm{P} \geq$ $\mathrm{Mg}>\mathrm{S}>\mathrm{Mn}>\mathrm{Fe}>\mathrm{B}>\mathrm{Zn}>\mathrm{Cu}$. A relação N/K foi de 2,3 em folhas e de 1,4 em frutos, indicando que na fase em que a planta entra em produção aumenta a necessidade de $K$. Apesar de o $P$ ser o quarto e o quinto nutriente mais requerido (em frutos e folhas, respectivamente) pela cultura, esse elemento deve ser fornecido em maior quantidade do que o acumulado, devido à facilidade de sua adsorção no solo. De posse da estimativa de acúmulo de nutrientes nos frutos, infere-se que o pinhãomanso extrai elevada quantidade de nutrientes na colheita e, se não adequadamente adubado, pode levar ao empobrecimento do solo ao longo dos anos de cultivo.
\end{abstract}

Termos de indexação: adubação, Jatropha curcas L., nutrição mineral, oleginosas.

\footnotetext{
(1) Recebido para publicação em agosto de 2007 e aprovado em julho de 2008.

${ }^{(2)}$ Pesquisador, Embrapa Agroenergia, Parque Estação Biológica - PqEB s/noo CEP 70770-901 Brasília (DF). E-mail: bruno.laviola@embrapa.br

(3) Professor Adjunto do Departamento de Fitotecnia, Universidade Federal de Viçosa - UFV. Av. P.H. Rolfs s s/nº CEP 36570-000 Viçosa (MG). E-mail: lasdias@ufv.br
} 


\title{
SUMMARY: NUTRIENT CONCENTRATION IN Jatropha curcas L. LEAVES AND FRUITS AND ESTIMATED EXTRACTIONAT HARVEST
}

\begin{abstract}
Knowledge about the nutritional demand of a crop is essential for fertilizer recommendation. In this study nutrient concentration and accumulation in leaves and fruits of Jatropha curcas $L$. were measured and nutrient extraction by fruits harvested between the $1^{\text {st }}$ and $4^{\text {th }}$ year of cultivation were estimated. The study was carried out at the Paraiso Farm, iin Viçosa, Minas Gerais State. Four leaf and fruit samples, each one containing 15 leaves or 20 fruits were randomly collected from an area with 6.000 Jatropha curcas L. trees, all planted in April 2006, at a spacing of $4 \times 2 \mathrm{~m}$. Expanded leaves between the $6^{\text {th }}$ and $8^{\text {th }}$ leaf below the inflorescence were collected for analysis. Only normal leaves (without signs of nutritional deficiency, insect attack and/or diseases) were used. Fruits were collected at maturity (yellow husk). The sampled leaves and fruits were washed, dried, weighed, and had their nutrient content analyzed. The nutrient concentration in Jatropha curcas $L$. leaves were ranked in the following order: $N>C a>K>M g>P>S>M n$ $>\mathrm{Fe}>\mathrm{B}>\mathrm{Zn}>\mathrm{Cu}$; and in the fruits the order was: $\mathrm{N}>\mathrm{K}>\mathrm{Ca}>\mathrm{P} \geq \mathrm{Mg}>\mathrm{S}>\mathrm{Mn}>\mathrm{Fe}>\mathrm{B}$ $>\mathrm{Zn}>\mathrm{Cu}$. The N/K ratio was 2.3 in the leaves and 1.4 in fruits, which indicates that $K$ requirements increase when the plant begins fruit production. Although $P$ is only the $4^{\text {th }}$ (in fruits) and the $5^{\text {th }}$ (in leaves) most demanded nutrient by the crop, it should be supplied in higher amounts than those accumulated in leaves and fruits due to soil adsorption. This study suggests that high nutrient amounts are extracted at Jatropha curcas $L$. harvest, thus the crop requires adequate fertilization to avoid soil exhaustion along the successive cultivation years.
\end{abstract}

Index terms: fertilization, Jatropha curcas L., mineral nutrition.

\section{INTRODUÇÃO}

O pinhão-manso (Jatropha curcas L.) é uma espécie perene e monóica, pertencente à família das Euforbiáceas, a mesma da mamona (Ricinus sp.), mandioca (Manhiot sp.) e seringueira (Hevea spp.). Acredita-se que a Jatropha seja originária da América Central, porém vegeta espontaneamente em diversas regiões do Brasil (Heller, 1996; Beltrão, 2005). É um arbusto de crescimento rápido, caducifólico, que pode atingir mais de $5 \mathrm{~m}$ de altura. Os frutos são do tipo cápsula ovóide, com 1,5 a 3,0 cm de diâmetro, trilocular, contendo via de regra três sementes, sendo uma semente por lóculo. As sementes têm de 1,5 a $2,0 \mathrm{~cm}$ de comprimento e 1 a $1,3 \mathrm{~cm}$ de largura, apresentam teor de óleo variando entre 33 e $38 \%$ e representam entre 53 e $79 \%$ do peso do fruto (Saturnino et al., 2005; Dias et al., 2007).

Quando plantado no princípio da estação chuvosa, o pinhão-manso inicia a produção de frutos já no primeiro ano de cultivo, embora atinja o seu clímax produtivo a partir do quarto ano, com capacidade produtiva potencial por mais de 40 anos. De acordo com informações mais atuais, a planta produz, em média, 100, 500, 2.000 e $4.000 \mathrm{~g} \mathrm{planta}^{-1}$ de sementes no primeiro, segundo, terceiro e quarto anos de cultivo, respectivamente (Tominaga et al., 2007). Dependendo do espaçamento, a produtividade pode passar dos $6.000 \mathrm{~kg} \mathrm{ha}^{-1}$ de sementes. Com essa produtividade, é possível produzir mais de $2.000 \mathrm{~kg} \mathrm{ha}^{-1}$ de óleo. No entanto, com o melhoramento genético e aprimoramento do sistema de produção, acredita-se que o pinhãomanso possa produzir acima de $4.000 \mathrm{~kg} \mathrm{ha}^{-1}$ de óleo.

O pinhão-manso é considerado uma cultura rústica, adaptada às mais diversas condições edafoclimáticas, que sobrevive bem em condições de solos marginais de baixa fertilidade natural (Arruda et al., 2004; Saturnino et al., 2005; Dias et al., 2007). Contudo, para se obter alta produtividade de frutos, a planta exige solos férteis e com boas condições físicas. Logo, a correção da acidez e da fertilidade do solo é decisiva para se obter sucesso e lucratividade nessa cultura.

Os resultados de pesquisas com a cultura do pinhão-manso são ainda incipientes e preliminares. Todavia, vem se observando extraordinária expansão da área cultivada com essa espécie. Embora não haja ainda dados estatísticos para todo o Brasil, o exemplo de Viçosa, município da Zona da Mata mineira, é bem representativo. A microrregião desse município cultiva, atualmente, 1.030 ha com pinhão-manso, implantado a partir de outubro de 2005. Esse crescimento de área plantada tem gerado expressiva demanda por informações, principalmente no que se refere às necessidades nutricionais da planta.

A recomendação de adubação de uma cultura depende das demandas nutricionais das plantas para os crescimentos vegetativo e reprodutivo (Laviola et al., 2007). Também deve ser levada em consideração a eficiência de aproveitamento dos adubos aplicados e a fração de nutrientes suprida pelo solo (Prezotti, 2001). 
A composição química tanto quanto o acúmulo de nutrientes em folhas e frutos são informações imprescindíveis para conhecer as exigências nutricionais de uma planta. Posteriormente, essas informações podem servir como subsídio para estimar a quantidade dos nutrientes a ser fornecida às plantas por meio da adubação.

O objetivo deste trabalho foi avaliar a composição e o acúmulo de macro e micronutrientes em folhas e frutos, bem como estimar a extração de nutrientes pela produção de frutos, visando subsidiar a recomendação de adubação para pinhão-manso.

\section{MATERIAL E MÉTODOS}

O estudo foi realizado na fazenda Paraíso, em Viçosa, MG (latitude $20^{\circ} 45^{\prime}$ sul, longitude $42^{\circ} 51^{\prime}$ oeste e altitude de $651 \mathrm{~m}$ ). Utilizou-se um talhão com 6.000 plantas de pinhão-manso, implantado em abril de 2006 em espaçamento de 4,0 x 2,0 m. O solo do talhão foi classificado como Latossolo VermelhoAmarelo distrófico (Quadro 1).

O pinhão-manso foi plantado em covas com dimensões de 0,4 x 0,4 x 0,4 m, utilizando-se a seguinte adubação no plantio: $100 \mathrm{~g}$ de calcário, $100 \mathrm{~g}$ do formulado 06-30-06 e $5 \mathrm{dm}^{3}$ de esterco de curral. Posteriormente, foram feitas adubações de cobertura com duas aplicações de 50 g/planta do formulado 2000-20, em setembro e novembro de 2006. Em janeiro de 2007 foi feita mais uma adubação mineral com $100 \mathrm{~g} /$ planta do formulado 20-05-20, e orgânica, com $2 \mathrm{dm}^{-3} /$ planta de esterco de curral.

Para o estudo foi realizada a coleta aleatória de quatro amostras de folhas e de frutos na área total, sendo cada uma composta por 15 folhas ou 20 frutos. Para as amostras de folhas, foram coletados limbos foliares expandidos, localizados entre a sexta e a oitava folha abaixo da inflorescência. A amostragem privilegiou folhas normais, sadias, sem deficiência nutricional aparente ou ataque de pragas e, ou, doenças. Os frutos foram coletados no estádio de maduro, com a casca amarela.

O material vegetal amostrado foi lavado em água deionizada e seco em estufa de circulação forçada de ar a $70^{\circ} \mathrm{C}$, até peso constante. Após esse processo, as amostras foram pesadas e moídas em moinho tipo Wiley, passadas em peneira de malha de 0,841 $\mathrm{mm}$ e submetidas a análises químicas.

Para determinação dos teores de $\mathrm{P}, \mathrm{K}, \mathrm{Ca}, \mathrm{Mg}, \mathrm{S}$, $\mathrm{Fe}$, Mn e Zn, o material vegetal, seco e moído, foi submetido à digestão nitroperclórica (Johnson \& Ulrich, 1959). O P foi determinado pelo método de redução do fosfomolibdato pela vitamina $\mathrm{C}$, modificado por Braga \& Defelipo (1974). O K foi determinado por fotometria de chama. $\mathrm{Ca}, \mathrm{Mg}, \mathrm{Fe}, \mathrm{Mn}$ e $\mathrm{Zn}$ foram quantificados por espectrofotometria de absorção atômica (AOAC, 1975), e S, por turbidimetria do sulfato (Jackson, 1958). O B foi analisado após digestão das amostras por via seca (calcinação em mufla a $550{ }^{\circ} \mathrm{C}$ ) e determinado por colorimetria pelo método da AzometrinaH (Bingham, 1982). Para determinação dos teores de $\mathrm{N}$, o material vegetal foi submetido à digestão sulfúrica (Jackson, 1958), sendo o nutriente quantificado de acordo com o método descrito por Bremner (1965).

$\mathrm{O}$ acúmulo de macro (mg/fruto) e micronutrientes ( $\mu \mathrm{g} /$ fruto) foi calculado pelas seguintes expressões:

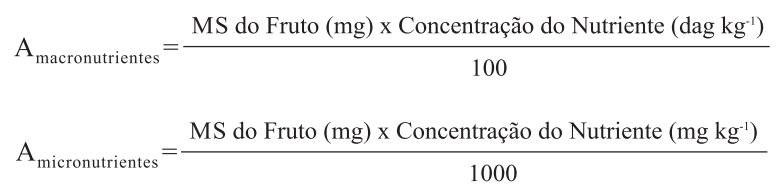

De posse dos valores de acúmulo de nutrientes em frutos de pinhão-manso, estimou-se a extração de nutrientes (EEN) por eles, por meio da expressão:

$$
\mathrm{EEN}=\mathrm{NF} \mathrm{X} \mathrm{A}
$$

em que $\mathrm{NF}=$ número de frutos produzidos; e $\mathrm{A}=$ acúmulo de nutriente por fruto.

$\mathrm{O} \mathrm{NF}$ foi estimado de acordo com a produção esperada de sementes por planta:

Quadro 1. Características químicas e físicas do solo da área experimental

\begin{tabular}{|c|c|c|c|c|c|c|c|}
\hline MO & $\mathrm{pH}\left(\mathrm{H}_{2} \mathrm{O}\right)$ & $\mathbf{P}$ & $\mathbf{K}$ & $\mathrm{Ca}^{2+}$ & $\mathrm{Mg}^{2+}$ & $\mathrm{Al}^{3+}$ & CTC \\
\hline $\mathrm{g} \mathrm{kg}^{-1}$ & & $-\mathrm{mg} \mathrm{dm}^{-3}$ & - & 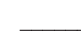 & - & $\mathrm{cmol}_{\mathrm{c}} \mathrm{dm}^{-3}$ & - \\
\hline 15 & 6,2 & 21,4 & 400 & 3,7 & 1,0 & 0 & 8,2 \\
\hline V & $\mathbf{S}$ & Premanescente & $\mathrm{Zn}$ & $\mathrm{Fe}$ & Mn & $\mathrm{Cu}$ & B \\
\hline$\%$ & $\mathrm{mg} \mathrm{dm} \mathrm{m}^{-3}$ & $\mathrm{mg} \mathrm{L}^{-1}$ & 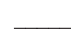 & 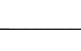 & $\mathrm{mg} \mathrm{dm}^{-3}$ & & - \\
\hline 70 & 29 & 56 & 10,1 & 37,5 & 76,9 & 2,3 & 0,9 \\
\hline
\end{tabular}

pH em $\mathrm{H}_{2} \mathrm{O}$ : relação 1:2,5. CTC: capacidade de troca de cátions. $\mathrm{P}, \mathrm{K}, \mathrm{Fe}, \mathrm{Zn}, \mathrm{Mn}, \mathrm{Cu}$ : extrator $\mathrm{Mehlich}^{-1}$. $\mathrm{Ca}^{2+}, \mathrm{Mg}^{2+}, \mathrm{Al}^{3+}$ : $\mathrm{KCl}$ $1 \mathrm{~mol} \mathrm{~L}{ }^{-1} \cdot \mathrm{H}+\mathrm{AL}$ : método $\mathrm{Ca}(\mathrm{OAc})_{2} 0,5 \mathrm{~mol} \mathrm{~L}^{-1}, \mathrm{pH}$. Matéria orgânica: C orgânico x 1,724-Walkley Black. 


$$
\mathrm{NF}=\frac{\mathrm{PS} \times \mathrm{F}}{\mathrm{PF}}
$$

em que PS = produção esperada de sementes por planta; $\mathrm{F}$ = fator de conversão de sementes para frutos, considerando-se que um fruto seco é composto de $36,5 \%$ de casca e $63,5 \%$ de sementes, sendo essas proporções determinadas previamente; e $\mathrm{PF}=$ peso de um fruto seco a $11,5 \%$ de umidade, considerando peso médio de $2,85 \mathrm{~g}_{\text {fruto }}{ }^{-1}$.

Para estimar a extração de nutrientes de acordo com a idade da planta, consideraram-se produções esperadas (PS) de 100, 500, 2.000 e 4.000 g planta $^{-1}$ de sementes no primeiro, segundo, terceiro e quarto anos de cultivo do pinhão-manso, respectivamente (Tominaga et al., 2007).

\section{RESULTADOS E DISCUSSÃO}

O pinhão-manso possui alto teor de nutrientes em seus tecidos (Quadros 2 e 3), apresentando a seguinte ordem de acúmulo no limbo foliar: $\mathrm{N}>\mathrm{Ca}>\mathrm{K}>\mathrm{Mg}>$ $\mathrm{P}>\mathrm{S}>\mathrm{Mn}>\mathrm{Fe}>\mathrm{B}>\mathrm{Zn}>\mathrm{Cu}$. Já em frutos maduros, observou-se a seguinte ordem de acúmulo de nutrientes: $\mathrm{N}>\mathrm{K}>\mathrm{Ca}>\mathrm{P} \geq \mathrm{Mg}>\mathrm{S}>\mathrm{Mn}>\mathrm{Fe}>\mathrm{B}$ $>\mathrm{Zn}>\mathrm{Cu}$. Nota-se que existem algumas particularidades em relação ao requerimento de macro e micronutrientes para formação das folhas e frutos, principalmente em relação à exigência de $\mathrm{Ca}$. É importante ressaltar que o pinhão-manso foi plantado em um solo de média a alta fertilidade (Quadro 1).

Por um lado, a ordem no acúmulo de nutrientes pelas folhas foi similar àquela observada por Saturnino et al. (2005), com apenas ligeiras diferenças no teor de alguns nutrientes. Por outro lado, os teores foliares de macronutrientes foram superiores às observadas por Beltrão et al. (2007); estes coletaram todas as folhas de uma planta de 100 dias para análise química. Neste trabalho foram avaliadas apenas folhas de ramos com inflorescência. A padronização dos órgãos amostrados é importante para fazer comparações e o diagnóstico nutricional de plantas. Nesse sentido, para se realizar amostragem foliar, sugere-se a coleta de folhas recémexpandidas, entre a sexta e a oitava folha abaixo da inflorescência. A vantagem de efetuar a amostragem de folhas em ramos com inflorescência é a de se avaliar o status nutricional da planta prematuramente, antes que os frutos se tornem drenos. Assim, se porventura a planta apresentar alguma deficiência nutricional nessa época, é possível corrigir o problema antes que este cause prejuízo à formação dos frutos e, conseqüentemente, à produção de grãos.

$\mathrm{O} \mathrm{N}$ foi o nutriente requerido em maior quantidade para formação das folhas, bem como para suprir as demandas metabólicas dos frutos. O pinhão-manso é uma planta que apresenta alta taxa de crescimento, sendo o $\mathrm{N}$ essencial para a assimilação do $\mathrm{C}$ e formação de novos órgãos na planta (Taiz \& Zeiger, 2004). Comparado às outras plantas, como cafeeiro (Laviola et al., 2006), goiabeira (Haag et al., 1993) e laranjeira (Mattos Jr. et al., 2003), o pinhão-manso apresentou maior teor de $\mathrm{N}$ nos frutos.

A relação N/K foi de 2,3 nas folhas e de 1,4 nos frutos, indicando que na fase em que a planta entra em produção há aumento do seu requerimento por K. Este nutriente possui papel importante na formação dos frutos, atuando no transporte de fotoassimilados no floema (Marschner, 1995). A deposição de biomassa no fruto é acompanhada, necessariamente, pelo acúmulo de $\mathrm{K}$. Além disso, este é um nutriente requerido na ativação de diversas enzimas essenciais à síntese de compostos orgânicos, entre eles o amido (Marschner, 1995; Marenco \& Lopes, 2005).

Apesar de o $\mathrm{P}$ estar entre o quarto e o quinto nutriente mais requerido pela cultura (Quadros $2 \mathrm{e}$ 3), ele é muito limitante, sobretudo na fase inicial de crescimento do pinhão-manso. Além de os solos brasileiros apresentarem baixo teor natural de P, este nutriente é rapidamente fixado pela fração argila, constituída, principalmente, por óxidos de $\mathrm{Fe}$ e Al, (Novais \& Smyth, 1999). Em alguns resultados de pesquisas constata-se que nos primeiros anos de cultivo o pinhão é muito responsivo à adubação fosfatada (Silva et al., 2007; Santos et al., 2007).

Quadro 2. Teores de macro e micronutrientes em folhas e frutos de pinhão-manso

\begin{tabular}{|c|c|c|c|c|c|c|c|c|c|c|c|}
\hline & $\mathbf{N}$ & $\mathbf{P}$ & $\mathbf{K}$ & $\mathbf{C a}$ & Mg & $\mathbf{S}$ & $\mathrm{Zn}$ & $\mathbf{F e}$ & Mn & $\mathrm{Cu}$ & B \\
\hline & \multicolumn{6}{|c|}{$\mathrm{g} \mathrm{kg}^{-1}$} & \multicolumn{5}{|c|}{$-\mathrm{mg} \mathrm{kg}^{-1}$} \\
\hline Folhas & 31,4 & 2,8 & 13,7 & 19,0 & 4,8 & 1,1 & 22,70 & 150,5 & 314,5 & 10,0 & 29,2 \\
\hline DP & 1,4 & 0,1 & 1,3 & 3,2 & 0,4 & 0,1 & 3,30 & 9,40 & 37,99 & 0,82 & 0,50 \\
\hline CV (\%) & 44,8 & 60,7 & 97,8 & 170,9 & 77,9 & 111,8 & 15,19 & 6,24 & 12,08 & 8,16 & 1,71 \\
\hline Frutos & 21,0 & 4,1 & 14,9 & 4,7 & 3,9 & 0,7 & 19,50 & 43,00 & 95,75 & 10,75 & 23,50 \\
\hline $\mathrm{DP}$ & 2,5 & 0,3 & 1,5 & 0,5 & 0,2 & 0,2 & 1,73 & 2,45 & 14,66 & 0,50 & 2,64 \\
\hline CV (\%) & 120,1 & 77,1 & 100,9 & 114,6 & 57,2 & 260,8 & 8,88 & 5,69 & 15,31 & 4,65 & 11,25 \\
\hline
\end{tabular}

DP: desvio-padrão. CV: coeficiente de variação. 
Assim, o fornecimento de $\mathrm{P}$ para o pinhão-manso, sobretudo nos primeiros anos de cultivo, deve ser em maior quantidade que o acumulado pela planta. À medida que a planta cresce, a área de exploração do sistema radicular se expande e a eficiência de recuperação de P no solo aumenta (Prezotti, 2001).

O Ca foi o segundo nutriente mais exigido para a formação das folhas e o terceiro mais acumulado pelos frutos. Isso demonstra que a cultura é exigente em $\mathrm{Ca}$, sendo importante que o solo apresente teores adequados desse nutriente para não limitar seu crescimento e sua produção. De modo geral, é ideal que o teor de $\mathrm{Ca}$ no solo seja mantido na faixa adequada, ou seja, acima de $3,5 \mathrm{cmol}_{\mathrm{c}} \mathrm{dm}^{-3}$, considerando uma relação de 4 a 3:1 com o Mg (Alvarez V. et al., 1999).

O B e Ca são indispensáveis à germinação do grão de pólen, ao crescimento do tubo polínico e, conseqüentemente, à fecundação da flor (Marschner, 1995). Entre os micronutrientes mais limitantes (B, $\mathrm{Zn}$ e $\mathrm{Cu}$ ), o B foi o mais requerido. O fornecimento inadequado deste nutriente pode contribuir para uma redução de produtividade, devido a um menor pegamento da florada.

As exigências de nutrientes minerais são menores nos dois primeiros anos de cultivo, aumentando rapidamente após o terceiro ano de implantação da cultura (Quadros 4 e 5). Esta é uma fração do que deve ser aplicado anualmente à lavoura, pois devem ser consideradas também a quantidade de nutrientes alocados na biomassa vegetativa e a quantidade de nutrientes que deixa de ser absorvida pela planta, em razão das diferentes taxas de recuperação dos diversos nutrientes do solo (Prezotti, 2001).

Considerando um espaçamento de $4 \times 2 \mathrm{~m}$ com 1.250 planta ha-1, a extração de nutrientes pelos frutos no quarto ano de cultivo corresponderia a uma retirada de $146,2,65,4$ e $124,38 \mathrm{~kg} \mathrm{ha}^{-1}$ de $\mathrm{N}, \mathrm{P}_{2} \mathrm{O}_{5}$ e $\mathrm{K}_{2} \mathrm{O}$, respectivamente. Esses valores mostram a elevada quantidade de nutrientes extraída do solo com a colheita dos frutos, que, se não adequadamente adubado, pode levar ao seu empobrecimento ao longo dos anos de cultivo. Somente a estimativa da extração de $\mathrm{N}$ pela colheita de frutos corresponde a 3,65 vezes a recomendação de $\mathrm{N}$ para a cultura da mamona, mandioca e girassol (CFSEMG, 1999a,b; Nogueira \& Gomes, 1999).

$\mathrm{Na}$ formulação das recomendações de adubação para a cultura, é importante considerar que os nutrientes minerais acumulados pelos frutos são exportados da área de cultivo pela colheita, sendo necessária a reposição integral desses elementos pelas

Quadro 3. Acúmulo de macro e micronutrientes em folhas e frutos de pinhão-manso

\begin{tabular}{|c|c|c|c|c|c|c|c|c|c|c|c|}
\hline & $\mathbf{N}$ & $\mathbf{P}$ & $\mathbf{K}$ & $\mathrm{Ca}$ & Mg & $\mathbf{S}$ & $\mathrm{Zn}$ & $\mathbf{F e}$ & Mn & $\mathrm{Cu}$ & B \\
\hline & \multicolumn{6}{|c|}{$\mathrm{mg} /$ folha } & 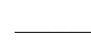 & 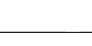 & \multicolumn{3}{|l|}{$\mu \mathrm{g} /$ folha } \\
\hline Follha & 36,47 & 3,31 & 15,85 & 22,36 & 5,61 & 1,31 & 25,46 & 175,2 & 368,8 & 11,71 & 34,01 \\
\hline DP & 3,16 & 0,32 & 1,37 & 5,62 & 0,88 & 0,19 & 5,40 & 19,81 & 74,35 & 2,08 & 3,91 \\
\hline \multirow[t]{2}{*}{ CV (\%) } & 8,67 & 9,73 & 8,70 & 25,16 & 15,70 & 14,83 & 21,22 & 11,31 & 20,15 & 17,82 & 11,47 \\
\hline & \multicolumn{6}{|c|}{$\mathrm{mg} /$ fruto } & \multicolumn{5}{|c|}{$\mu \mathrm{g}$ fruto ${ }^{-1}$} \\
\hline Fruto & 53,01 & 10,35 & 37,57 & 12,01 & 9,79 & 1,75 & 49,34 & 108,4 & 241,8 & 27,15 & 59,05 \\
\hline DP & 7,34 & 1,11 & 4,13 & 1,87 & 1,00 & 0,43 & 6,62 & 8,05 & 41,36 & 2,47 & 4,45 \\
\hline CV (\%) & 13,86 & 10,79 & 11,00 & 15,57 & 10,28 & 24,72 & 13,42 & 7,42 & 17,10 & 9,10 & 7,54 \\
\hline
\end{tabular}

DP: desvio-padrão. CV: coeficiente de variação.

Quadro 4. Estimativa de extração de macronutrientes pela produção de frutos, de acordo com a produção esperada de sementes nos respectivos anos de cultivo

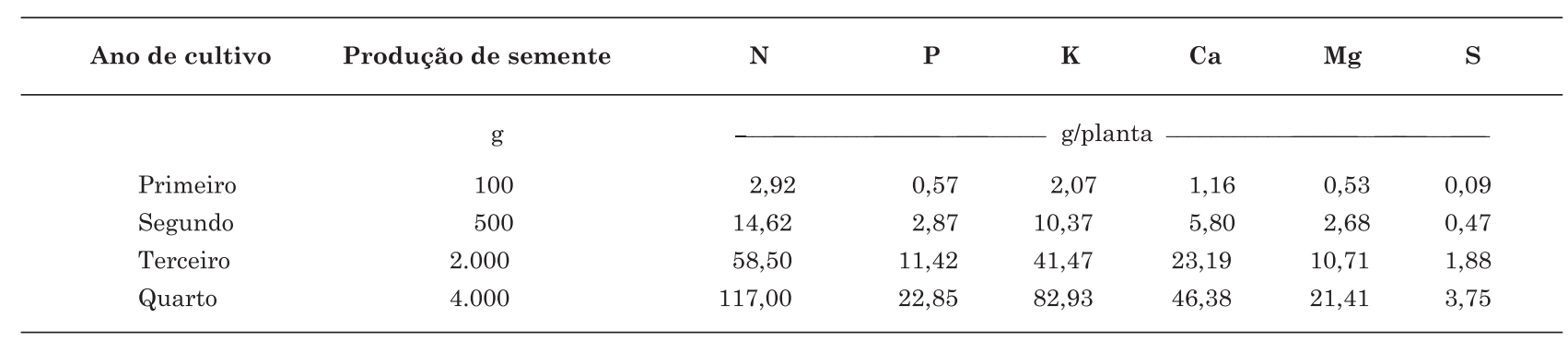


Quadro 5. Estimativa de extração de micronutrientes pela produção de frutos, de acordo com a produção esperada de sementes nos respectivos anos de cultivo

\begin{tabular}{|c|c|c|c|c|c|c|}
\hline Ano de cultivo & Produção de semente & $\mathrm{Zn}$ & $\mathbf{F e}$ & Mn & $\mathrm{Cu}$ & B \\
\hline \multicolumn{7}{|c|}{$\mathrm{mg} /$ planta } \\
\hline Primeiro & 100 & 2,72 & 5,98 & 13,34 & 1,50 & 3,26 \\
\hline Segundo & 500 & 13,61 & 29,91 & 66,72 & 7,49 & 16,29 \\
\hline Terceiro & 2.000 & 54,45 & 119,64 & 266,88 & 29,97 & 65,17 \\
\hline Quarto & 4.000 & 108,89 & 239,29 & 533,76 & 59,93 & 130,35 \\
\hline
\end{tabular}

práticas de adubação. Por outro lado, parte dos nutrientes acumulados pela planta é reciclada no sistema solo-planta, devido à queda de folhas no inverno. Com a decomposição das folhas no solo, os nutrientes são liberados para reabsorção do sistema radicular. Além disso, os nutrientes contidos nas folhas, que são móveis no floema, podem ser remobilizados para pontos de crescimento e, ou, frutos, antes que as folhas caiam (Epstein \& Bloom, 2006). Além disso, ainda deve ser observada a proporção do que a planta necessita de cada nutriente. A proporção encontrada no acúmulo de $\mathrm{N}: \mathrm{P}_{2} \mathrm{O}_{5}: \mathrm{K}_{2} \mathrm{O}$ pelos frutos de pinhão-manso foi de 2,23:1:1,90 (Quadro 4). Como uma primeira aproximação, sugere-se, para as práticas de adubação da cultura, a partir do segundo ano de cultivo, o uso dos formulados cujas proporções de $\mathrm{N}: \mathrm{P}_{2} \mathrm{O}_{5}: \mathrm{K}_{2} \mathrm{O}$ estejam próximas às encontradas nos frutos.

\section{CONCLUSÕES}

1. O acúmulo de nutrientes pelas folhas obedeceu à seguinte ordem: $\mathrm{N}>\mathrm{Ca}>\mathrm{K}>\mathrm{Mg}>\mathrm{P}>\mathrm{S}>\mathrm{Mn}>\mathrm{Fe}$ $>\mathrm{B}>\mathrm{Zn}>\mathrm{Cu}$; nos frutos, a ordem encontrada foi: $\mathrm{N}$ $>\mathrm{K}>\mathrm{Ca}>\mathrm{P} \geq \mathrm{Mg}>\mathrm{S}>\mathrm{Mn}>\mathrm{Fe}>\mathrm{B}>\mathrm{Zn}>\mathrm{Cu}$.

2. A colheita dos frutos de pinhão-manso extrai elevada quantidade de nutrientes do solo; se estes não forem adequadamente repostos pela adubação, poderá ocorrer empobrecimento do solo ao longo dos anos de cultivo.

\section{LITERATURA CITADA}

ALVAREZ V., V.H.; NOVAIS, R.F.; BARROS, N.F.; CATARUTTI, R.B. \& LOPES, A.S. Interpretação dos resultados das análises de solos. In: RIBEIRO, A.C.; GUIMARÃES, P.T.G. \& ALVAREZ V., V.H., eds. Recomendações para o uso de corretivos e fertilizantes em Minas Gerais, $5^{\mathrm{a}}$ Aproximação. Viçosa, MG, Comissão de Fertilidade do Solo do Estado de Minas Gerais, 1999. p.25-32.
ARRUDA, F.P.; BELTRÃO, N.E.M.; ANDRADE, A.P.; PEREIRA, W.E. \& SEVERINO, L.S. Cultivo de pinhãomanso (Jatropha curcas L.) como alternativa para o semiárido nordestino. R. Bras. Oleag. Fibrosas, 8:789-799, 2004.

ASSOCIATION OF OFFICIAL ANALYTICAL CHEMISTS AOAC. Official methods of analysis. 12.ed. Washington, 1975. 1094p.

BELTRÃO, N.E.M.; SEVERINO, L.S.; SUINAGA, F.A.; VELOSO, J.F.; JUNQUEIRA, N.; FEDELIS, M.; GONÇALVES, N.P.; SATURNINO, H.M.; ROSCOE, R.; GAZZONI, D.; DUARTE, M.A.; DRUMOND, M.A. \& ANJOS, J.B. Recomendação técnica sobre o plantio de pinhão-manso no Brasil. Disponível em:< http:// www.cpao.embrapa.br/portal/noticias/ Position \%20Paper.pdf > Acesso em: 16 de julho de 2007.

BELTRÃO, N.E.M.; LIMA, R.L.S.; SEVERINO, L.S.; SAMPAIO, L.R.; SOFIATTI, V. \& LEÃO, A.B. Crescimento e acúmulo de nutrientes pelo pinhão-manso sob interferência de plantas daninhas. In: CONGRESSO BRASILEIRO DE PLANTAS OLEAGINOSAS, ÓLEOS, GORDURAS E BIODIESEL, 4., Varginha, 2007. Anais. Lavras, Universidade Federal de Lavras 2007. p.1892-1900.

BELTRÃO, N.E.M. Agronegócio das oleaginosas no Brasil. Inf. Agropec., 26:44-78, 2005.

BINGHAM, F.T. Boron. In: PAGE, A.L., ed. Methods of soil analysis: Chemical and microbiological properties. Madison, American Society of Agronomy, 1982. p.431447. (Serie Agronomy, 9)

BRAGA, J.M. \& DEFELIPO, B.V. Determinação espectrofotométrica de $\mathrm{P}$ em extratos de solo e material vegetal. $\mathrm{R}$. Ceres, 21:73-85, 1974.

BREMNER, J.M. Total nitrogen. In: BLACK, C.A., ed. Methods of soil analysis. Madison, American Society of Agronomy, 1965. p.1149-1178.

COMISSÃO DE FERTILIDADE DO SOLO DO ESTADO DE MINAS GERAIS - CFSEMG. Mamona. In: RIBEIRO, A.C.; GUIMARÃES, P.T.G. \& ALVAREZ V., V.H., eds. Recomendações para o uso de corretivos e fertilizantes em Minas Gerais, $5^{a}$ Aproximação. Viçosa, MG, 1999a. p.311. 
COMISSÃO DE FERTILIDADE DO SOLO DO ESTADO DE MINAS GERAIS - CFSEMG. Girassol. In: RIBEIRO, A.C.; GUIMARÃES, P.T.G. \& ALVAREZ V., V.H., eds. Recomendações para o uso de corretivos e fertilizantes em Minas Gerais, $5^{a}$ Aproximação. Viçosa, MG, 1999b. p.310.

DIAS, L.A.S.; LEME, L.P.; LAVIOLA, B.G.; PALLINI FILHO, A.; PEREIRA, O.L.; CARVALHO, M.; MANFIO, C.E.; SANTOS, A.S.; SOUSA, L.C.A.; OLIVEIRA, T.S. \& DIAS, D.C.F.S. Cultivo de pinhão-manso (Jatropha curcas L.) para produção de óleo combustível. Viçosa, MG, 2007. v.1. $40 \mathrm{p}$.

EPSTEIN, E. \& BLOOM, A.J. Nutrição mineral de plantas: Princípios e perspectivas. 2.ed. Londrina, Planta, 2006. 401p.

HAAG, H.P.; MONTEIRO, F.A. \& WAKAKURI, P.Y. Frutos de goiaba (Psidium guayava L.): Desenvolvimento e extração de nutrientes. Sci. Agric., 50:413-418, 1993.

HELLER, J. Physic nut (Jatrhopha curcas L.). Promiting the conservation and use of underutilized and neglected crops 1. IBPGR 161. Roma, IBPGR, 1996. 66p.

JACKSON, M.L. Soil chemical analysis. New Jersey, Prentice Hall, 1958. 498p.

JOHNSON, C.M. \& ULRICH, A. Analytical methods for use in plants analyses. Los Angeles, University of California, 1959. v.766. p.32-33.

LAVIOLA, B.G.; MARTINEZ, H.E.P.; SOUZA, R.B.; SALOMÃO, L.C.C. \& CRUZ, C.D. Acúmulo de macronutrientes em frutos de cafeeiros em viçosa-MG. In: SIMPÓSIO DE PESQUISA DOS CAFÉS DO BRASIL 5., Águas de Lindóia, 2007. Anais... Águas de Lindóia, 2007. CD-ROM.

LAVIOLA, B.G.; MARTINEZ, H.E.P.; SOUZA, R.B. \& ALVAREZ V., V.H. Dinâmica de N e K em folhas, flores e frutos de cafeeiro arábico em três níveis de adubação. Biosci. J., 22:33-47, 2006.

MARENCO, R.A. \& LOPES, N.F. Fisiologia vegetal: Fotossíntese, respiração, relações hídricas e nutrição mineral. Viçosa, MG, Universidade Federal de Viçosa, 2005. 451p.
MARSCHNER, H. Mineral nutrition of higher plants. 2.ed. New York, Academic Press, 1995. 889p.

MATTOS JR., D.; QUAGGIO, J.A.; CANTARELLA, H. \& ALVA, A.K. Nutrient contento of biomass components of Hamlin sweet orange trees. Sci. Agric., 60:155-160, 2003.

NOGUEIRA, F.D. \& GOMES, J.C. Mandioca. In: RIBEIRO, A.C.; GUIMARÃES, P.T.G. \& ALVAREZ V., V.H., eds. Recomendações para o uso de corretivos e fertilizantes em Minas Gerais, $5^{\mathrm{a}}$ Aproximação. Viçosa, MG, Comissão de Fertilidade do Solo do Estado de Minas Gerais CFSEMG, 1999. p.312.

NOVAIS, R.F. \& SMYTH, T.J. Fósforo em solo e planta em condições tropicais. Viçosa, MG, Universidade Federal de Viçosa, 1999. 300p.

PREZOTTI, L.C. Fertilização do cafeeiro. In: ZAMBOLIM, L. Tecnologias de produção de café com qualidade. Viçosa, MG, Universidade Federal de Viçosa, 2001. p.607-615.

SANTOS, S.; FERREIRA Jr., E.J.; PIRES, B. \& NETTO, A.P.C. Efeito de diferentes adubações no desenvolvimento inicial de mudas de pinhão-manso (Jatropha curcas L.). In: CONGRESSO BRASILEIRO DE PLANTAS OLEAGINOSAS, ÓLEOS, GORDURAS E BIODIESEL, 4., Varginha, 2007. Anais... Lavras, Universidade Federal de Lavras, 2007. p.547-554.

SATURNINO, H.M.; PACHECO, D.D.; KAKIDA, J.; TOMINAGA, N. \& GONÇALVES, N.P. Cultura do pinhãomanso (Jatropha curcas L.). Inf. Agropec., 26:44-78, 2005.

SILVA, J.T.A.; COSTA, E.L.; SILVA, I.P. \& NETO, A.M. Adubação do pinhão-manso (Jatropha curcas L.) com nitrogênio e fósforo. In: CONGRESSO BRASILEIRO DE PLANTAS OLEAGINOSAS, ÓLEOS, GORDURAS E BIODIESEL, 4, Varginha, 2007, Anais. Lavras, Universidade Federal de Lavras, 2007. p.1316-1320.

TAIZ, L. \& ZEIGER, E. Fisiologia vegetal. 3.ed. Porto Alegre, Artmed, 2004. 719p.

TOMINAGA, N.; KAKIDA, J. \& YASUDA, E.K. Cultivo de pinhão-manso para produção de biodiesel. Viçosa, MG, CPT, 2007. 220p. 